\title{
Four remarks on multiparticle correlations
}

\author{
Andrzej Bialas
}

Institute of Nuclear Physics, PAS, Krakow, Radzikowskiego 152, Poland

DOI: http://dx.doi.org/10.5689/UA-PROC-2010-09/15

Personal comments are made on (a) Long-range correlations, including (i) multiplicity distributions and (ii) forward-backward correlations; (b) Short-range correlations, including (i) Balance functions, and (ii) BE correlations and intermittency.

\section{Negative Binomial Distribution}

It seems that we shall be forced again and again to discuss the negative binomial distributions (NBD). As shown e.g. by ALICE experiment [1], it still describes reasonably well the data in the interval $|\eta|<1$ even at $7 \mathrm{TeV}$.
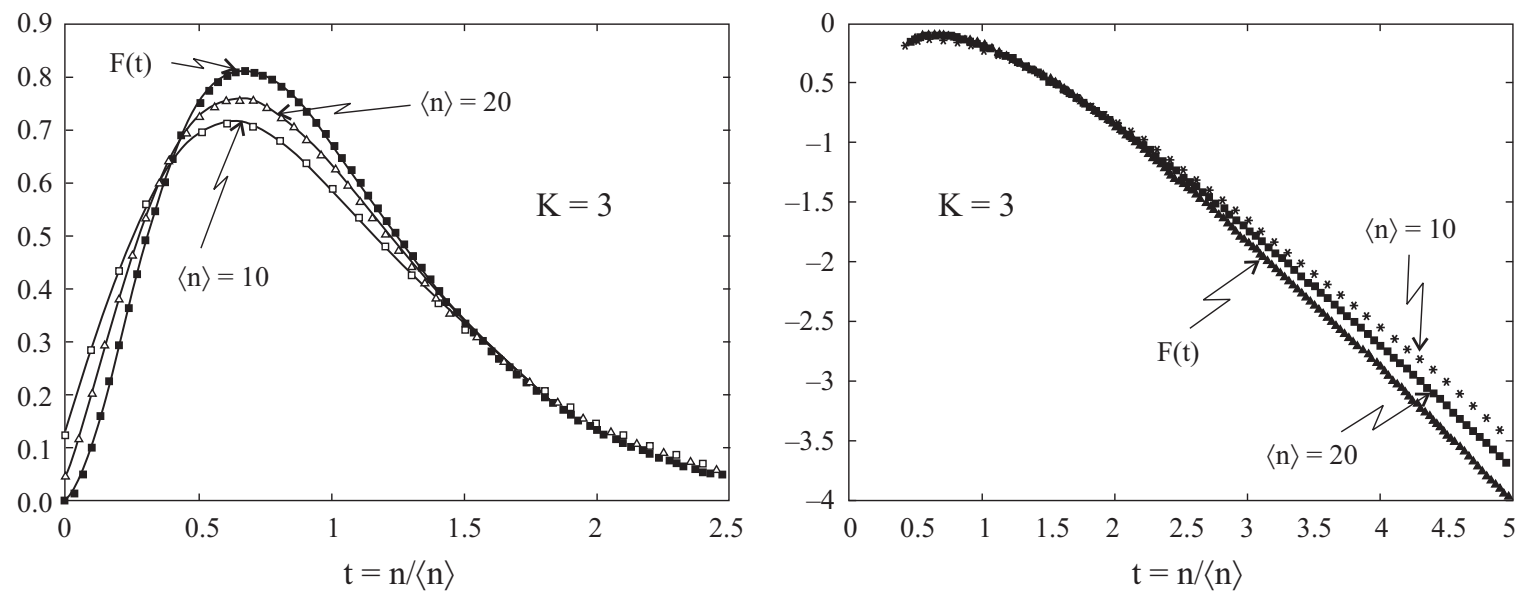

Figure 1: Dependence of the Negative Binomial Distribution on the average multiplicity. Left panel: linear scale; Right panel: log scale.

The negative binomials, first introduced into high-energy physics by Alberto Giovannini [2] belong to the general class of the distributions of the form

$$
P(n)=\int F(t) d t e^{-t \bar{n}} \frac{(t \bar{n})^{n}}{n !} ; \quad \int F(t) d t=1 ; \quad \int F(t) t d t=1 .
$$


In case of NBD $F(t)$ is the $\Gamma$ distribution:

$$
F(t)=\frac{k^{k}}{\Gamma(k)} t^{k-1} e^{-k t}
$$

Eq (1) describes a superposition of various sources, each one emitting a bunch of entirely uncorrelated particles.

This interpretation invites one to treat the parameter $t$ as a measure of the "intensity" of a source, the function $F(t)$ representing the (relative) distribution of source intensities. One may then ask the question how one can uncover the function $F(t)$, from the observed multiplicity distribution, i.e. how to inverse the transformation (1). In the limit of very large average multiplicity $\bar{n}$ the result is well known [3]

$$
\bar{n} P(n) \rightarrow F(t=n / \bar{n})
$$

One can verify, however, that, when applied to negative binomial distribution, this prescription gives a good approximation only for indeed large multipliities. This is shown in the figure 1, where $\bar{n} P(n)$ is plotted for various average multiplicities. One sees that this commonly used approximation must be treated with a great caution. Some improvements can of course be devised [4].

It is also well known since the works of Giovannini, Van Hove and Ekspong [5], that the negative binomial distribution can be also understood as an independent emission of "clans", decaying with the probability dstribution

$$
p(n)=\frac{k}{<N>} \frac{1}{n}\left[\frac{<n>}{<n>+k}\right]^{n} ; \quad n \geq 1
$$

I would like to observe that this "clan" interpretation can be formulated more generally, if we allow that clans are not necessarily independently emitted. This follows from the observation [6] that the generating function of NBD can be written in the form

$$
\Phi(z) \equiv\left[1+\frac{\bar{n}}{k}(1-z)\right]^{-k}=\left[1+\frac{<N>}{K}[1-\phi(z)]\right]^{-K}
$$

with

$$
\phi(z)=1+\frac{K}{<N>}-\frac{K}{<N>}\left[1+\frac{<n>}{k}(1-z)\right]^{k / K}
$$

These formulae describe the emission of clans according to negative binomial distribution (characterized by the parameter $K$ ) and clan decay distribution in the form

$$
p(n)=\frac{k}{<N>}\left[1+\frac{<n>}{k}\right]^{k / K} \frac{(1-k / K) \ldots(n-1-k / K)}{n !}\left[\frac{<n>}{k+<n>}\right]^{n} .
$$

For $K \rightarrow \infty$ one recovers (4). 


\section{Forward-backward correlations}

It is now widely recognized that forward-backward (f-b) correlations in rapidity give information about the early stages of the collision. Indeed, it is difficult to imagine how such correlations may appear at the late stages of the development of the produced system when particles are separated by large distances due to longitudinal expansion. Just after the collision, however, the system is small, so that its various parts may communicate with each other and therefore correlations can extend through the whole system.

Studies of f-b correlations have a long history [7]. They obtain a really new significance at the advent of the LHC data. The new point is energy.

One of the essencial issues in discussion of mechanisms of particle production is the question if the produced particles "remember" the colliding projectiles, their energy, momentum and quantum numbers. Obviously the answer depends on the kinematic region we are considering. Close to the fragmentation region, the influence of the projectile on the produced particle spectrum is naturally expected. In the central rapidity region, far from the projectile fragmentation, the question remains open. On the theoretical side there is no consensus and various models give different answers.

There is no space here to review the models ${ }^{1}$, so let me simply say that -with respect to this question- they may be divided into three categories. In the first one, originated from the famous Landau and Feynman papers $[9,10]$, particles produced in the central rapidity region are detached from the projectiles. Thus the source of particles is symmetric with respect to $y=0$. In the second class, like the wounded nucleon model [11], particles are produced by quasiindependent emisssion from the two colliding objects. In this case particles in the central region come from two sources, this time naturally asymmetric ones $[12,13,14]$. There is of course also a third class which combines the two pictures, a typical example being the dual-parton model $[15]$.

These various mechanisms can be tested (and verified) by studying the f-b correlations. The essential point is that correlations for one symmetric source are generally much stronger than those induced by two asymmetric sources $[8,16]$.

It would thus be very interesting to measure them at LHC. Several points are of particular interest:

(i) UA5 data at $200 \mathrm{GeV}$ show clearly [16] that the asymmetric scenario is preferred. But the new LHC data show a rapid increase of particle multiplicity in the central region. Is this increase due to a new, symmetric component, as suggested by some models [15], or is it just fast increase of production from asymmetric sources?

(ii) It will be very interesting to compare the $p p$ and $A A$ data and thus learn to what extent the original correlations are modified (washed out?) by the evolution of the QGP. In view of the recent CMS data [17], it is clearly also worthwhile to investigate the sector of large multiplicities.

(iii) The $200 \mathrm{GeV}$ data suggest that $\mathrm{f}$-b correlations do not extend through the whole rapidity available at this energy but only through a part of it [16]. It is important to determine how this region varies with energy. This, unfortunately, may require larger acceptance than presently available.

Going outside of the hadronic collisions, it is clear thet studies of f-b correlations in $e^{+} e^{-}$ and deep-inelastic collisions are also of great interest.

\footnotetext{
${ }^{1}$ There is an excellent review in [7], see also [8]. Here I only quote some examples.
} 
To end this part, let me mention that a systematic method of investigating the f-b correlations in symmetric processes was recently developped [8]. The relations were derived between the factorial cumulants in one (say, forward) bin and the joint factorial cumulants in two (forward and backward) bins. They allow to distinguish between differents mechanisms. E.g. if only symmetric sources are present the relations are particularly simple:

$$
f_{k l}=f_{k+l}
$$

where $f_{k l}$ is the joint factorial cumulant in two intervals and $f_{k+l}$ is the factorial cumulant in one of the intervals.

The first two relations give

$$
\begin{gathered}
<n_{F} n_{B}>=<n_{F}\left(n_{F}-1\right)>; \\
<n_{F}\left(n_{F}-1\right) n_{B}>=<n_{F}\left(n_{F}-1\right)\left(n_{F}-2\right)>.
\end{gathered}
$$

\section{Balance functions revisited}

New data from STAR on balance functions in $p p$ and $A u A u$ collisions [18] confirmed the important qualitative conclusions which were formulated in the first publication [19]: the rapidity balance functions measured in heavy ion collisions are narrower than those in "elementary" $p p$ collisions (see also $[20,21,22]$ ). The observation that balance functions in (pseudorapidity) are narrow, has an immediate and important consequence: it shows that the charges are created at the late stage of the collision, just before freeze-out [23, 24]. This of course eliminates models in which charges appear soon after the collision took place [18, 24].

A more difficult is the problem why the width observed in central heavy ion collisions is smaller than that in peripheral and in $p p$ collisions. In other words, what is the source of the new short range correlations revealed by this observation. As discussed in [24], one can introduce these additional correlations by supplementing the blast wave model [25] with the requirement that pairs of charges are created in relatively small domains of space.

It was also observed few years ago [26] that narrowing of balance functions can be explained -in a natural way- by clustering of gluons in the plasma. Following the information that the charges appear only at the very end, it is assumed that the QGP at the end of its evolution is made of uncorrelated isotropic clusters of gluons ("glue droplets") which decay into (constituent) $u \bar{u}, d \bar{d}$ or $s \bar{s}$ pairs. To create a charged particle one needs to coalesce [27] a quark and an antiquark from two different gluon droplets. The basic reason for narrowing of the balance function width (in pseudorapidity) is the well-known fact that the dispersion of the average of two independent variables is by factor $\sqrt{2}$ smaller than the dispersion of each of them. Correction due to transverse flow and limited acceptace can be estimated but they do not change this basic conclusion.

The new STAR paper gives data on balance functions in momentum space. They confirm the smaller width of balance function in central $A u A u$ collisions as compared to peripheral and $p p$ collisions. It was therefore interesting to look what are the consequences of this new data for the model of $[26]^{2}$.

The STAR data show that the observed balance functions are highly anisotropic. This is in apparent contradition with the picture of isotropic glue droplets. It turns out, however,

\footnotetext{
${ }^{2}$ The pseudorapidity distributions depend solely on the particle production angle and are thus only marginally sensitive to the momentum distribution.
} 
that acceptance corrections are large enough to explain this contradiction. In a rather crude estimate, using $<\delta_{\text {long }}>=0.19 \mathrm{GeV}$ as an input, one obtains

$$
<\delta_{\text {side }}>=0.284 \mathrm{GeV} ; \quad<\delta_{\text {out }}>=0.126 \mathrm{GeV}
$$

to be compared with experimentally observed

$$
<\delta_{\text {side }}>=0.28 \pm 0.01 \mathrm{GeV} ; \quad<\delta_{\text {out }}>=0.11 \pm 0.01 \mathrm{GeV},
$$

a very reasonable result.

It remains an interesting question if these glue droplets appear only at the last stage of the QGP development (close to freeze-out) or rather are a permanent feature of the system of gluons. In the second case they may clearly have important impact on the evolution of the plasma.

\section{Intermittency and HBT correlations}

Few years ago Csorgo et al. [28, 29] have had a great idea to study the HBT correlations in the Lund Linked Dipole Chain model [30, 31] of multigluon emissions which describes in detail the fractal structure built in successive jet decays. Since the Lund model links directly the momentum and ordinary space, this analysis allowed to study quantitatively the relation between the HBT correlations and intermittency [32] in a QCD-inspired model.
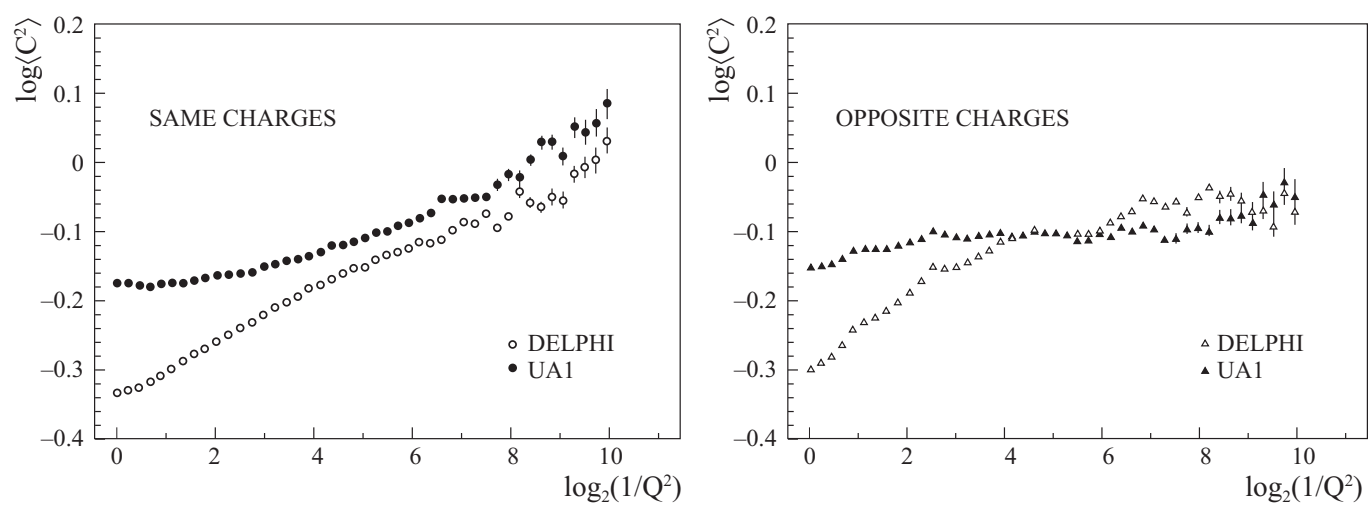

Figure 2: Correlation coefficient $C^{(2)}$ versus $1 / Q^{2}[\mathrm{GeV}]$ in $\log -\log$ scale.

It was shown [28] that the fractal structure associated with jet decay gives the two-particle HBT correlation function in the form of the Levy stable distribution:

$$
C_{2}=1+\lambda e^{-|q R|^{\alpha}}
$$

where $q$ is the momentum difference of the two identical particles and $R$ is a scale factor ${ }^{3}$. Furthermore, it is argued in [28] that the exponent $\alpha$ which satisfies the inequality

$$
0 \leq \alpha \leq 2
$$

\footnotetext{
${ }^{3}$ The corresponding source distribution in configuration space has a power law tail $|x|^{-\alpha-1}$. The scale $R$ determines the region at small $|x|$ where the power law breaks down so that the distribution does not blow up at $|x|=0$.
} 
is related to the anomalous dimension of QCD:

$$
\alpha=\sqrt{\frac{3 \alpha_{s}}{2 \pi}}
$$

where $\alpha_{s}$ is the strong coupling constant.

In [29] the form (12) was compared to UA1 and NA22 data and shown to be adequate. The fitted values of $\alpha$ were used to evaluate $\alpha_{s}$.

At this point I would like to make a simple observation, related to my earlier discussion of the fluctuating sources [34]: If the scale parameter $R$ fluctuates according to the distribution $F(R) d R,(12)$ is tranformed into

$$
C_{2}(q)=1+\lambda \int d R F(R) e^{-|q R|^{\alpha}}
$$

and this expression may take form very different from (12), as illustrated by the following two examples.

As the first example we take the distribution ${ }^{4}$

$$
F(R) d R=\frac{2 \omega}{\pi} e^{-\omega^{2} x^{-2} / \pi} \frac{d x}{x^{2}}
$$

with $x=R^{\alpha / 2}$. This gives

$$
C_{2}(q)=1+\lambda \int \frac{2 \omega}{\pi} e^{-\omega^{2} x^{-2} / \pi} \frac{d x}{x^{2}} e^{-|q|^{\alpha} x^{2}}=1+\lambda e^{-v|q r|^{\alpha / 2}}
$$

with $r^{\alpha / 2} v=2 \omega / \sqrt{\pi}$. One sees that after this transformation the Levy parameter $\alpha$ changes into $\alpha / 2$.

As the second example, let consider the fluctuations of the parameter $R^{\alpha}$ in the form of the $\Gamma$ distribution:

$$
F\left(R^{\alpha}\right) d R^{\alpha}=\frac{k^{k}}{\Gamma(k)} R^{\alpha(k-1)} e^{-k R^{\alpha}} d R^{\alpha}
$$

The result is

$$
C_{2}==1+\lambda\left[1+\frac{|q R|^{\alpha}}{k}\right]^{-k}
$$

a typical result for the intermittency-driven HBT correlation [34].

The two transformations (17) and (19) can be of course combined into a single one.

It remais an open question if the idea presented here can be related to the fluctuating string tension [33]. If these two effects have a common origin, we should have the relation

$$
\sigma \sim \frac{1}{R^{\alpha}}
$$

whe $\sigma$ is the string tension.

\footnotetext{
${ }^{4}$ What follows is a modified version of the argument used in [33].
} 
Thus we are forced to conclude that the condition of stability is not enough to determine the exact form of the correlation function, since it apparently depends on details of the system. Also the parameter $\alpha$ is most likely not universal.

Still, it is clear that the idea formulated in $[28,29]$ is of great value. First, it demonstrated how the fractal structure, inherent in parton cascades, can be quantitatively implemented into analysis of HBT phenomenon. Perhaps even more important is the observation that the obtained momentum distribution is valid not only in a limited (small) values of $q^{2}$ but in a broad region largely exceeding the interval where HBT phenomenon is best visible. This may be a possible explanation of the long-standing puzzle: why the experimentally observed "intermittency slope" does not change when $q^{2}$ goes beyond the HBT region [35]. The problem is illustrated in Figure 2, taken from [36]. One sees that the DELPHI data for like-sign particles follow approximately a single straight line (with the slope not largely different from that shown by unlike-sign particles at large $Q^{2}[37]$ ), although the physics at small $Q^{2}$ is apparently very different from that at $Q^{2} \approx 1 \mathrm{GeV}$.

\section{Acknowledgements}

I would like to thank Krzysztof Fialkowski and Edward Sarkissian-Grinbaum for encouragement and opportunity to present this talk. This investigation was supported in part by the grant $\mathrm{N}$ N202 125437 of the Polish Ministry of Science and Higher Education (2009-2012).

\section{References}

[1] K.Aamodt et al, ALICE coll., Eur. Phys. J. C68 (2010) 89; C68 (2010) 345.

[2] A.Giovannini, Nuovo Cim. A15 (1973) 543.

[3] Z.Koba, H.B.Nielsen and P.Olesen, Nucl.Phys. B40 (1972) 317.

[4] A. Bialas, Acta Phys. Pol. B41 (2010) 2163.

[5] A.Giovannini and L.Van Hove, Z.Phys. C30 (1986) 213; L.Van Hove and A.Giovannini, Acta Phys. Pol. B19 (1988) 495; G.Ekspong, Proc. XVI ISMD, Kiryat Anavim (1985), Ed. J,Grunhaus, Edition Frontieres, World Scientific, 1986, p. 309.

[6] A.Bialas, Acta Phys. Pol. B41 (2010) 1899.

[7] For a review, see W.Kittel and E.A.De Wolf, Soft Multihadron Dynamics, World Scientific (2005).

[8] A.Bialas and K.Zalewski, Phys. Rev. C82 (2010) 034911.

[9] L.Landau, Izv. Akad. Nauk Ser. Fiz. 17 (1953) 51.

[10] R.P. Feynman, Phys. Rev. Lett. 23 (1969) 1415.

[11] A.Bialas, M.Bleszynski and W.Czyz, Nucl.Phys. B111 (1976) 461; A.Bialas, W.Czyz and W.Furmanski, Acta Phys. Pol. B8 (1977) 585.

[12] A.Bialas and W. Czyz, Acta Phys. Pol. B36 (2005) 905.

[13] A. Bialas and A.Bzdak, Acta Phys. Pol. B38 (2007) 159; Phys. Lett. B649 (2007) 263; Phys. Rev. C77 (2008) 034908. For a review, see A.Bialas, J.Phys. G35 (2008) 044053.

[14] B.Anderson, G.Gustafson and B.Nilsson-Almqvist, Nucl.Phys. B281 (1987) 289.

[15] For a review, see A.Capella, U.Sukhatme, C.-I.Tan and J.Tran Thanh Van, Phys. Rep. 236 (1994) 225.

[16] A. Bzdak, Acta Phys. Pol. B41 (2010) 151; arXiv 0906.2858; Acta Phys. Pol. B40 (2009) 2029.

[17] CMS coll., JHEP 1009 (2010) 091.

[18] STAR coll., M.M. Aggarwal et al., Phys.Rev. C82 (2010) 024905. 
[19] STAR Collaboration, J.Adams et al., Phys. Rev. Lett. 90 (2003) 172301.

[20] B.I.Abelev et al., STAR coll., Phys. Lett. B690 (2010) 239.

[21] C.Alt et al., NA49 Coll., Phys. Rev C71 (2005) 034903.

[22] C.Alt et al., NA49 Coll., Phys. Rev C76 (2007) 024914.

[23] S.A.Bass, P.Danielewicz and S.Pratt, Phys. Rev. Lett. 85 (2000) 2689.

[24] S.Cheng et al., Phys. Rev. C69 (2004) 054906.

[25] See e.g., F.Retiere and M.Lisa, Phys. Rev. C70 (2004) 044907.

[26] A. Bialas, Phys. Lett. B579 (2004) 31; see also A.Bialas and J.Rafelski, Phys. Letters B633 (2006) 488.

[27] T.S.Biro, P.Levai and J.Zimanyi, Phys. Lett. B347 (1995) 6; J.Zimanyi, P. Levai, T.S.Biro, Heavy Ion Phys. 17 (2003) 205 and references quoted there; J.Pisut and N.Pisutova, Acta Phys. Pol. B28 (1997) 2817; R.Lietava, J.Pisut, Eur. Phys. J. C5 (1998) 135.

[28] T.Csorgo,S.Hegyi,T.Novak and W.A.Zajc, Acta Phys. Pol. B36 (2005) 329.

[29] T.Csorgo,S.Hegyi and W.A.Zajc, Eur.Phys.J. C36 (2004) 67.

[30] B.Andersson, G.Gustafson, J.Samuelson, Nucl.Phys. B463 (1996) 217, B467 (1996) 443.

[31] For a review, see B.Andersson, The Lund Model, Cambridge U. Press (Cambridge 1998).

[32] A.Bialas and R.Peschanski, Nucl.Phys. B273 (1986) 703; Nucl.Phys. B308 (1988) 857.

[33] A.Bialas, Phys. Letters B466 (1999) 301.

[34] A.Bialas, Acta Phys. Pol. B23 (1992) 561; AIP Conf.Proc. 828 (2006) 409.

[35] A.Bialas, Proc. XXVII Int. Conf. on High Energy Phys., Glasgow (1994). Ed. P.J.Bussey and I.G.Knowles, IOP Publishing, Bristol and Philadelphia (1995), p.1287.

[36] UA1 and DELPHI coll., F.Mandl and B.Buschbeck, Proc XXII ISMD, Santiago de Compostela 1992, Ed. A.Pajares, World Scientific (1993).

[37] W.Kittel, remark in the discussion after the talk. 